\title{
Multimodal imaging documentation of rapid evolution of retinal changes in handheld laser-induced maculopathy
}

\author{
Elona Dhrami-Gavazi 1,2,3, Winston Lee ${ }^{3}$, Chandrakumar Balaratnasingam,2,4, Larisa Kayserman5,
} Lawrence A. Yannuzzi ${ }^{1,2}$ and K. Bailey Freund ${ }^{1,2^{*}}$

\begin{abstract}
Purpose: To use multimodal imaging to document the relatively rapid clinical evolution of handheld laser-induced maculopathy (HLIM). To demonstrate that inadvertent ocular injury can result from devices mislabeled with respect to their power specifications.

Methods: The clinical course of a 17-year-old male who sustained self-inflicted, central macular damage from a 20-25 s direct stare at a red-spectrum, handheld laser pointer ordered from an internet retailer is provided. Retrospective review of multimodal imaging that includes fundus photography, fluorescein angiography, MultiColor reflectance, eye-tracked spectral domain optical coherence tomography (SD-OCT), fundus autofluorescence, and microperimetry is used to describe the evolving clinical manifestations of HLIM in the first 3 months.
\end{abstract}

Results: Curvilinear bands of dense hyperreflectivity extending from the outer retina and following the Henle fibers were seen on SD-OCT immediately after injury. This characteristic appearance had largely resolved by 2 weeks. There was significant non-uniformity in the morphological characteristics of HLIM lesions between autofluorescence and reflectance images. The pattern of lesion evolution was also significantly different between imaging modalities. Analysis of the laser device showed its wavelength to be correctly listed, but the power was found to be 102.5-105 mW, as opposed to the $<5 \mathrm{~mW}$ described on the label.

Conclusion: While the immediate SD-OCT characteristics are highly specific for handheld laser -induced maculopathy, this finding can undergo rapid resolution in the span of several days. In the absence of this finding, other multimodal imaging clues and a careful history may aid in recognizing this diagnosis. A greater awareness regarding inaccurate labeling on some of these devices could help reduce the frequency of this preventable entity.

Keywords: Handheld laser-induced maculopathy, Multimodal imaging

\section{Background}

Since the first report by Zamir et al. [1], a great number of additional cases of handheld laser-induced maculopathy (HLIM) have appeared in the ophthalmic and general medical literature. A recent increase in the frequency of these reports may be related to an increase in the availability of laser pointers intended for the public. These

\footnotetext{
*Correspondence: kbfnyf@aol.com

1 Vitreous-Retina-Macula Consultants of New York, 460 Park Avenue, 5th Floor, New York, NY 10022, USA

Full list of author information is available at the end of the article
}

devices appear to be easily obtained through loosely regulated internet-based retailers. Various case reports and case series have characterized the clinical manifestations of this entity and have described the patterns that distinguish self-inflicted from accidental occurrences [1-8]. Self-inflicted cases of HLIM may manifest characteristic linear streaks on color photographs and other imaging modalities which will typically show a vertical pattern in the superior macula. This finding may relate to a discomfort-induced Bell's phenomenon when staring at an intense light. Conversely, the accidental and peer-inflicted cases of HLIM typically appear as rounder 
lesions in the central macula [3]. Adolescent males are the typical demographic to present with HLIM and yellow/grey-colored lesions are commonly identified in the central and superior macula in the immediate period following injury [3]. Characteristic curvilinear hyperreflective bands are also seen on spectral domain optical coherence tomography (SD-OCT) imaging during this period [2-4].

The temporal course of HLIM immediately following injury, has not been documented in depth, but chronological studies that have reported mid- and long-term findings have suggested that the clinical manifestations of this disease evolve at a rapid rate. It is therefore important to understand the temporal characteristics of laserinduced maculopathy in order to distinguish it from other entities that may present with similar clinical signs. We present a patient with HLIM whose disease course in the 3 months after injury was closely and frequently documented with multimodal imaging. This report provides new information about the natural course and multimodal imaging characteristics of HLIM.

\section{Case presentation}

A 17-year-old male was referred for evaluation of a 4 day history of decreased central vision in his right eye. He was under treatment for attention deficit hyperactivity disorder with $5 \mathrm{mg}$ per day dexmethylphenidate hydrochloride extended release but was otherwise healthy. Past ocular history and family ocular history were both unremarkable. During the evaluation, the patient was pointedly asked whether he had been exposed to a hand held laser pointer. Reluctantly, he admitted that his visual symptoms occurred immediately after staring directly at the light emitted from a red laser pointer for $20-25 \mathrm{~s}$. His father had purchased this device over the internet.

On examination, the patient was emmetropic with best-corrected visual acuity of 20/100 in his right eye and 20/20 in his left eye. The anterior segment, lens and vitreous were unremarkable in both eyes. Funduscopic examination of the right eye revealed several sharply demarcated lesions with an excavated appearance, yellowish borders, and grey-green centers (Fig. 1a). These lesions were arranged in a vertical fashion in the central

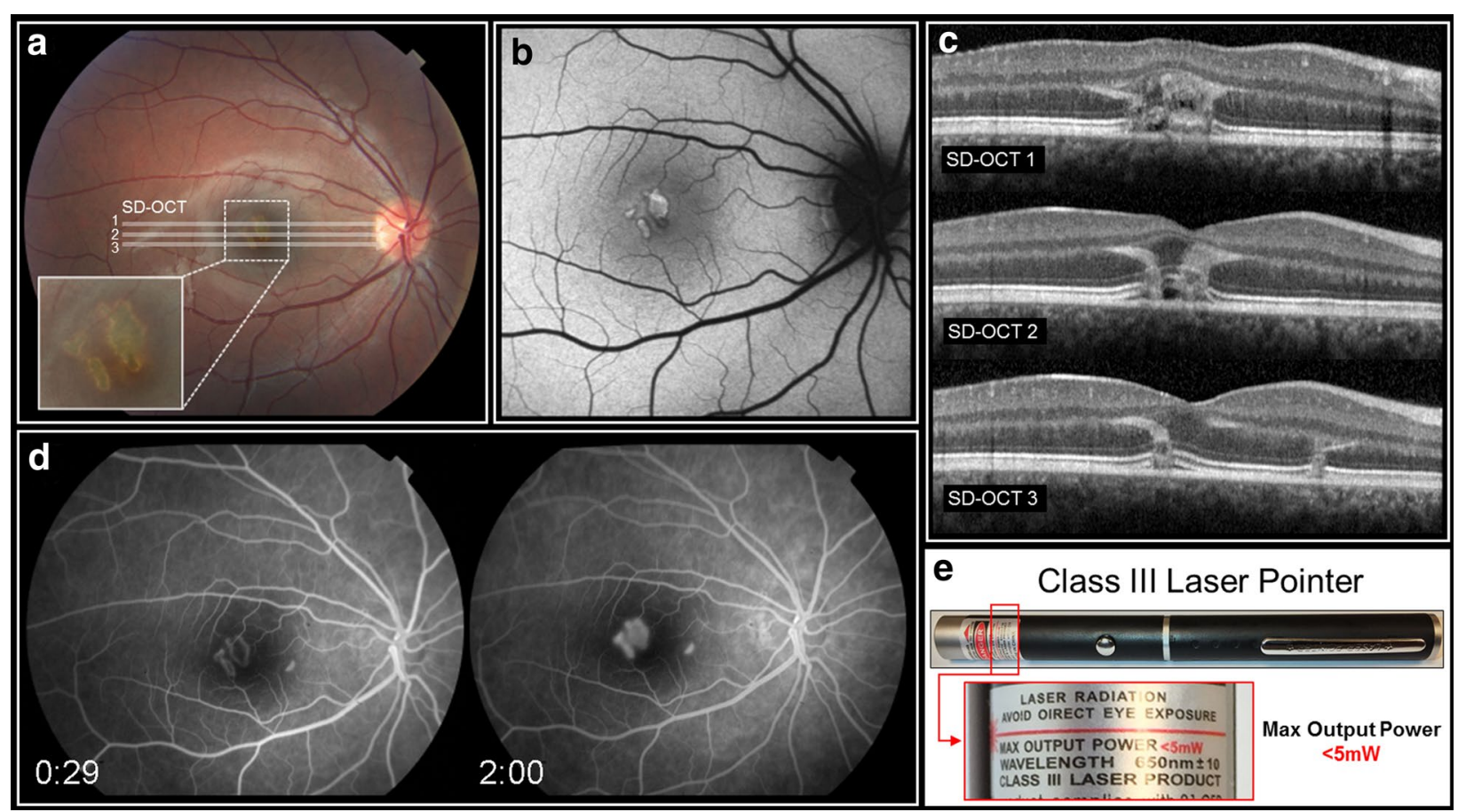

Fig. 1 Funduscopic findings at presentation as documented via multimodal imaging. a Color photograph of the right eye taken 1 day after the injury shows sharply demarcated, "excavated" green-grey lesions with yellow borders situated in a vertical-oblique fashion in the central macula. b Fundus autofluorescence taken 1 day after the injury shows central hyperfluorescence of the lesions with a thin surrounding rim of hyperautofluorescence. c Spectral domain optical coherence tomography images of three different sections taken 1 day after the injury show curvilinear bands of dense hyperreflectivity that extend from the interdigitation layer and ellipsoid zone of the photoreceptors upwards, ending at the level of the outer plexiform layer. The hyperreflective lesions appear to follow the Henle fibers. There are small hyporeflective cavities beneath the fovea. $\mathbf{d}$ Fluorescein angiography taken 1 day after the injury shows hyperfluorescence of the lesions with minimal leakage. An additional satellite lesion, not detected with color photos and autofluorescence, is evident nasal to the fovea. e Photograph of the device used in the injury with a magnified view (inset) of its label 

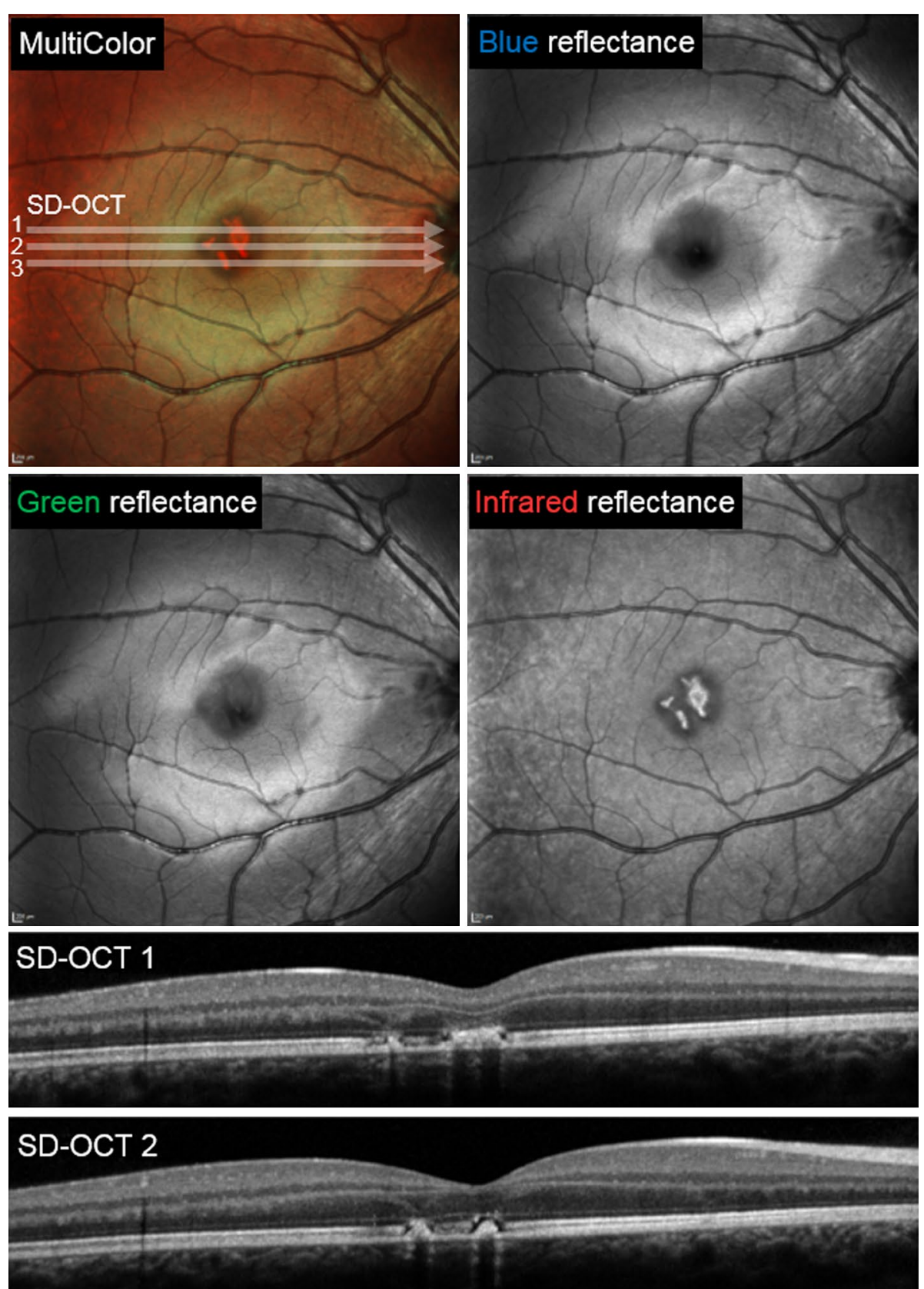

\section{SD-OCT 3}

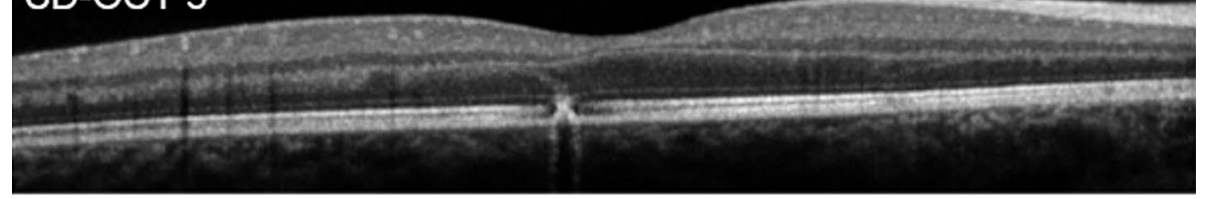

Fig. 2 MultiColor and spectral domain optical coherence tomography findings obtained 7 days following the injury. The lesions are not seen with blue-reflectance but become increasingly apparent with the longer green and infrared reflectance wavelengths. Note that while the multicolor image sharply depicts the borders of the lesions, it may misrepresent the true color of their center as seen on funduscopy. Three SD-OCT scans through the central macula show that the vertical curvilinear hyperreflective bands are attenuated 7 days after the initial injury 
macula. The remainder of the fundus appeared normal. The funduscopic examination of the left eye showed no abnormalities. Fundus autofluorescence showed lesions that co-localized with the lesion seen in the color photographs (Fig. 1b). Fluorescein angiography performed by the referring physician 1 day following the onset of symptoms showed hyperfluorescence of the central macular lesions with minimal leakage. An additional lesion, invisible on exam, was evident nasal to the fovea (Fig. 1d). This lesion was not observed on reflectance imaging or color photography. SD-OCT performed 1 day after the injury showed curvilinear bands of dense hyperreflectivity that extended from the interdigitation layer and ellipsoid zone of the photoreceptors upwards, ending at the level of the outer plexiform layer (Fig. 1c). The hyperreflective lesions appeared to follow the Henle fibers. There were small hyporeflective cavities beneath the fovea. MultiColor and SD-OCT findings taken 7 days following the onset of symptoms are shown in Fig. 2. The evolution of the SD-OCT findings was documented over 3 months of follow-up (Fig. 3). Eye-tracked SD-OCT line scans taken at 1 day, 4 days, 2 weeks, 1 month and 3 months after injury documented the rapid evolution of the vertical curvilinear bands of hyperreflectivity. At the most
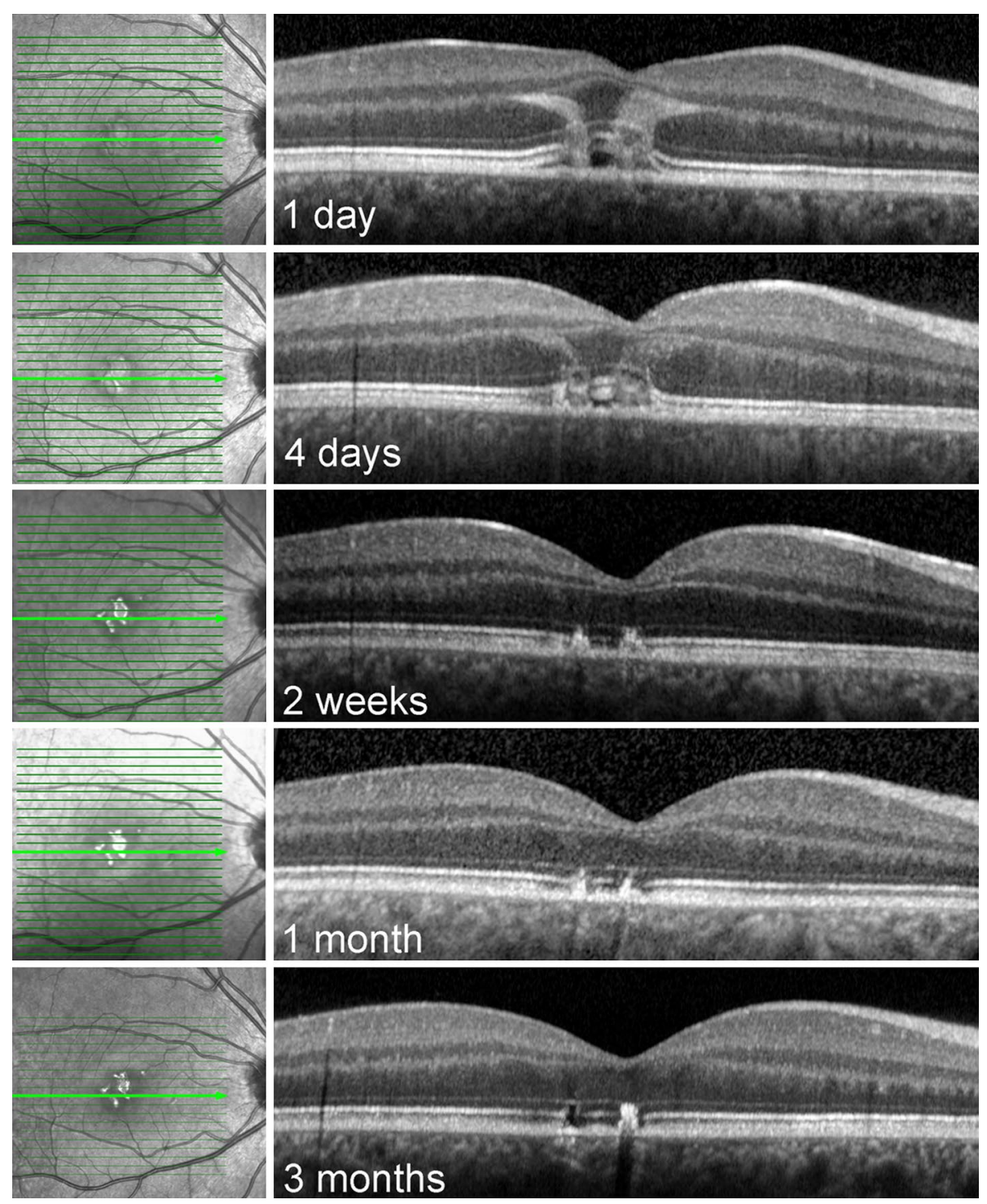

Fig. 3 Evolution of the spectral domain optical coherence tomography: findings over 3 months. Eye-tracked, subfoveal SD-OCT line scans of the right eye are shown. The scans were taken at 1 day, 4 days, 2 weeks, 1 month and 3 months after the onset of the patient's visual symptoms. Note how quickly the vertical curvilinear bands of hyperreflectivity resolve 
recent follow-up evaluation, 3 months following the initial injury, visual acuity in the right eye had improved to 20/30. Funduscopic examination of right eye showed central pigment hyperplasia with some surrounding depigmentation of the lesions in the macula (Fig. 4a). Fundus autofluorescence showed near normalization of the acute changes (Fig. 4b), but the size of the lesions on this modality was observably smaller than the infrared reflectance image (Fig. 4c). Infrared reflectance showed high reflectivity of the macular lesions. Microperimetry showed persistent central scotomas and slightly eccentric fixation (Fig. 4d).

We were able to obtain the device that caused the injury and measure its output. While the center wavelength was $663 \mathrm{~nm}$, approximating the label specification of $650 \pm 10 \mathrm{~nm}$, its power output was grossly mislabeled. The device had been labelled as having a maximum power output of $<5 \mathrm{~mW}$. However, evaluation of the device with two different optical power meters revealed values that were considerably higher than the label's specifications. With the original batteries, the power measured $85 \mathrm{~mW}$ (Newport 1918-R) and $90 \mathrm{~mW}$ (Thorlabs PM100). With new batteries, the power measured $102.5 \mathrm{~mW}$ (Newport 1918-R) and $105 \mathrm{~mW}$ (Thorlabs PM100) (Table 1). Thus, the laser pointer was incorrectly labeled as "Class III Laser Product" (Fig. 1d) when, as per the above measurements, it should have been classified as a Class IIIB laser, which requires a key switch and a safety interlock. The parents and the patient were counseled as to the hazards of this seemingly "harmless" laser pointer device.

\section{Conclusions}

With the increasing frequency of handheld laser induced maculopathy, its findings on multimodal imaging, particularly SD-OCT, the affected demographic, and the mid to long-term course of this injury have become more clearly defined [1,3-8]. The coexistence of the characteristic curvilinear bands on SD-OCT along with the other

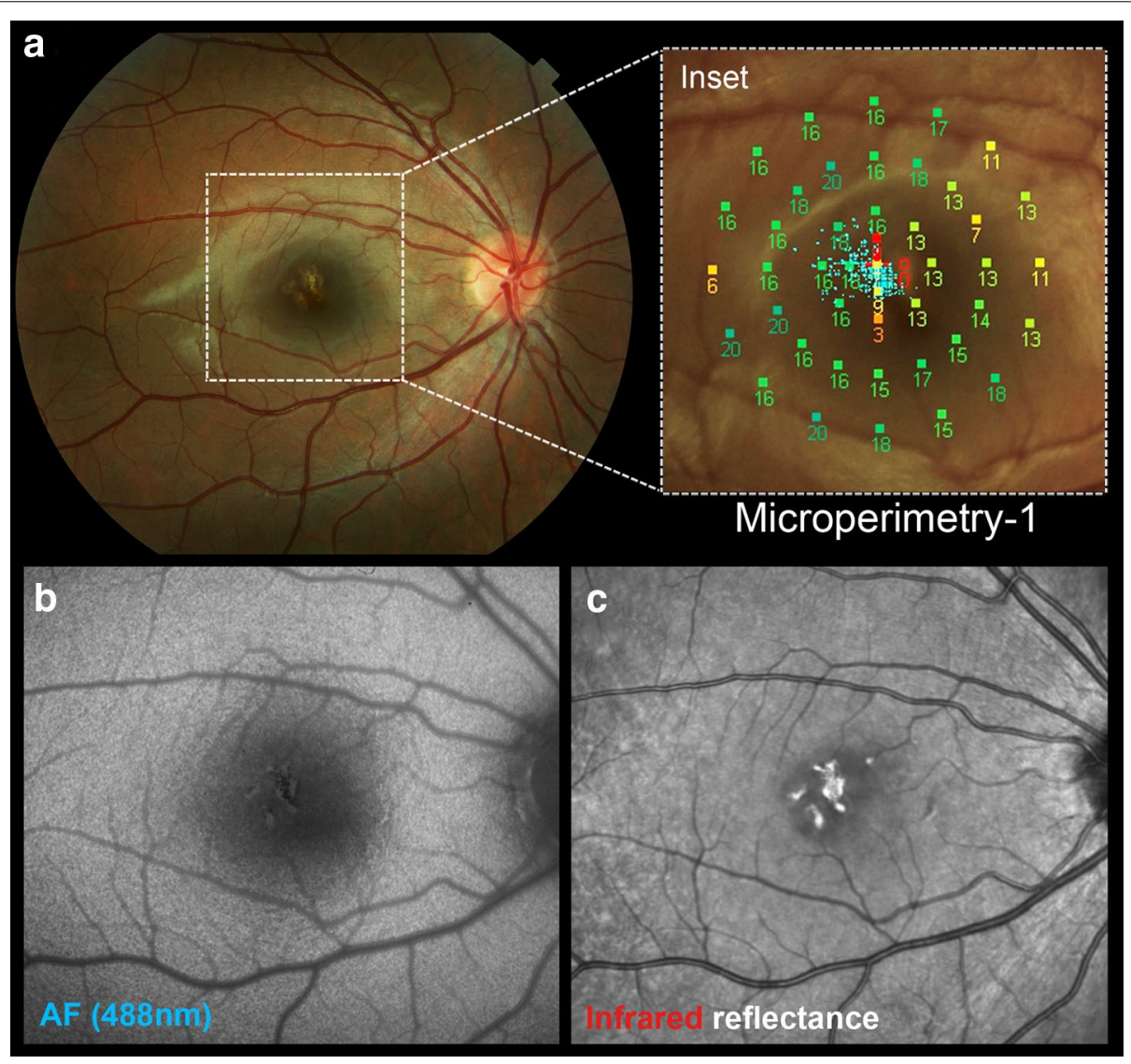

Fig. 4 The 3 month visit findings as documented by multimodal imaging. a Color photograph of the right eye shows that the lesions now demonstrate central pigment hyperplasia with some surrounding depigmentation. Inset Microperimetry shows persistent central scotomas and slightly eccentric fixation. Visual acuity is 20/30 in the right eye. b Fundus $(488 \mathrm{~nm}$ ) autofluorescence shows near normalization of the acute changes and underappreciation of the lesion boundaries in comparison with the infrared image. $\mathbf{c}$ Infrared reflectance image shows high reflectivity of the macular lesions 
Table 1 Handheld laser pointer optical power measurements and safety reclassification

\begin{tabular}{|c|c|c|c|}
\hline \multicolumn{4}{|l|}{ Wavelength (nm) } \\
\hline \multicolumn{2}{|l|}{ Device specification } & \multicolumn{2}{|l|}{$650 \pm 10$} \\
\hline \multicolumn{2}{|l|}{ Center wavelength (FWHM) } & \multicolumn{2}{|l|}{$663(661-664)$} \\
\hline \multicolumn{2}{|l|}{ Optical power (mW) } & Original batteries $(\mathrm{mW})$ & New batteries \\
\hline \multicolumn{2}{|l|}{ Device specification } & $<5$ & $<5 \mathrm{~mW}$ \\
\hline \multicolumn{2}{|l|}{ Newport 1918-R } & 85 & 102.5 \\
\hline \multicolumn{2}{|l|}{ Thorlabs PM100 } & 90 & 105 \\
\hline \multicolumn{2}{|l|}{ Safety classification } & \multicolumn{2}{|l|}{ Required precaution(s) } \\
\hline Device specification & Class III & \multicolumn{2}{|c|}{ "Caution" warning label on device } \\
\hline Measured & Class IIIB ${ }^{b}$ & \multicolumn{2}{|c|}{$\begin{array}{l}\text { Necessary safety components: key switch, a safety interlock dongle, a power indica- } \\
\text { tor, an aperture shutter, and an emission delay }\end{array}$} \\
\hline
\end{tabular}

FWHM full width at half maximum, $n m$ nanometer, $m W$ microWatt

a Also referred to as Class IIIA (ANSI Z136.1) or 3R (IEC 60825-1)

b Also referred to as Class IIIB (ANSI Z136.1) or 3B (IEC 60825-1)

findings observed in multimodal imaging is a pathognomonic pattern that, in tandem with history taking, serves to establish a prompt diagnosis. This case illustrates the temporal evolution of SD-OCT changes immediately following HLIM. It also illustrates the similarities and the discrepancies in lesion characteristics between different imaging modalities.

The recognition of the characteristic green-grey vertical lesions located in the central macula, particularly in young male patients complaining of acute to subacute vision loss, helps to establish this diagnosis. Our case demonstrates that the characteristic vertical curvilinear bands of hyperreflectivity visible in Henle's fiber layer resolved within 2 weeks. SD-OCT changes in the course of HLIM may therefore be a short-lived finding. The importance of an immediate pattern-recognition is paramount as it may eliminate the need for unnecessary and expensive medical evaluations. More importantly, correct diagnosis may prevent further damage from the same device, at times to the fellow eye (if the injury is initially unilateral) and complications including full-thickness macular hole, choroidal neovascularization, hemorrhage, and permanent deep, central scotomata. Other reports have demonstrated evidence of persistent OCT lesions beyond the 2 week period [3], and it is therefore likely that the temporal behavior of OCT findings is related to the wavelength and power of the laser device as well as the duration of laser exposure during the initial insult.

Previous reports by our group and others [2-7] have noted that, although strict regulations by the Food and Drug Administration and the American National Standards Institute govern the safe use of medical, research and industrial lasers, there is no regulatory body monitoring the use of the widely available, easily accessible and potentially mislabeled handheld laser pointers. As evidenced by this case, the power output of these devices can greatly exceed what is listed on the label (by nearly $100 \mathrm{~mW}$ in our case), and the laser mislabeling allows for the omission of any mandatory safety measures for the power it actually possesses such as key switches, safety interlocks and/or the recommended use of safety goggles.

In summary, this case shows the rapid evolution of SDOCT findings in HLIM. Awareness of these characteristic features can facilitate a prompt diagnosis of this entity even when the typical juxtafoveal vertical curvilinear opacities are absent on SD-OCT.

\section{Consent}

Written informed consent was obtained from the patient's parents for publication of this case report and any accompanying images. A copy of the written consent is available for review by the Editor-in-Chief of this journal.

\section{Abbreviations}

SD-OCT: spectral-domain optical coherence tomography; HLIM: handheld laser-induced maculopathy.

\section{Authors' contributions}

ED-G-acquisition of data, analysis and interpretation of images, conceptualization of manuscript, review of literature, drafting/editing of the report, preparation of image panels. WL-acquisition of data, drafting/editing of the report, preparation of image panels. CB - analysis and interpretation of images, editing of report. LK-acquisition of data, editing of report. LAY — conceptualization of manuscript, editing the report, analysis and interpretation of images, final approval of the report. KBF-conceptualization of manuscript, editing the report, analysis and interpretation of images, final approval of the report. All authors read and approved the final manuscript.

\section{Authors' information}

Elona Dhrami-Gavazi received her medical degree at the University of Tirana, Albania. She completed Ophthalmology residency training in Albania after 
which she completed a second Ophthalmology residency training at Columbia University in New York, serving as a chief resident in both trainings. She is currently a vitreoretinal fellow at Columbia University, Vitreous Retina-Macula Consultants of New York and Manhattan Eye Ear Throat Hospital, New York. She is a recipient of the Victor A. McCusick Research Fellowship Grant from the National Marfan Foundation.

Winston Lee received his master's degree from Columbia University and is currently a graduate research assistant at the Department of Ophthalmology, Columbia University Medical Center.

Chandrakumar Balaratnasingam received his medical degree and $\mathrm{PhD}$ at the University of Western Australia. He completed Ophthalmology training in Australia after which he completed fellowship vitreoretinal surgical training in Perth, Australia and Vancouver, Canada. He is currently a fellow at the Vitreous Retina-Macula Consultants of New York.

Larisa Kayserman obtained her BA in Chemistry at New York University, where she received the distinguished Founders Day Award. She graduated Cum Laude from the State University of New York at Downstate Medical School and achieved membership in the Alpha Omega Alpha Honor Society. She completed her Residency in Ophthalmology and served as a Chief Resident at SUNY Downstate/Kings County Medical Center, as well as a Fellowship in Retina-Vitreous Diseases and Surgery at SUNY Downstate/Kings County Medical Center.

Lawrence Yannuzzi is founder of VRMNY as well as vice-chairman and director of the LuEsther T. Mertz Retinal Research Center of the Manhattan Eye, Ear and Throat Hospital and the founder and president of The Macula Foundation, Inc. He has published over 400 scientific papers and 13 books and is the recipient of numerous awards, including an honorary doctorate by the University of Ancona, the Michelson Award for Retinal Vascular Disease, a Distinguished Alumnus Award by Boston University, the Henkind, Gass, and Patz Medals by The Macula Society, the Alcon Research Award, the Herman Wacker Award of the Club Jules Gonin, the Arthur J. Bedelle Award, the Retinal Research Award and the Gass Medal of the Retina Society, the Bietti Medal, the Pisart Award from the Lighthouse International, and the Lifetime Achievement Award by the American Academy of Ophthalmology.

K. Bailey Freund is a Clinical Professor of Ophthalmology at New York University School of Medicine and a senior partner at Vitreous Retina Macula Consultants of New York. He is on the Editorial Board of the journal Retina. He has authored over 225 peer-reviewed scientific manuscripts and numerous book chapters. He is a recipient of the Young Investigator Award from the Macula Society.

\section{Author details}

${ }^{1}$ Vitreous-Retina-Macula Consultants of New York, 460 Park Avenue, 5th Floor, New York, NY 10022, USA. ${ }^{2}$ The LuEsther T. Mertz Retinal Research Center, Manhattan Eye Ear and Throat Hospital, New York, NY, USA. ${ }^{3}$ Department of Ophthalmology, Edward S. Harkness Eye Institute, College of Physicians and Surgeons, Columbia University, New York, NY, USA. ${ }^{4}$ Department of Physiology and Pharmacology, Centre for Ophthalmology and Visual Sciences, Lions Eye Institute, University of Western Australia, Perth, Australia. ${ }^{5}$ Retina Consultants of New Jersey, New Jersey, NJ, USA.

\section{Acknowledgements}

The authors thank Caroline R. Baumal, M.D., for her invaluable assistance in obtaining the device specifications and measurements.

\section{Financial support}

LuEsther T. Mertz Retinal Research Center, Manhattan Eye, Ear and Throat Hospital, New York, NY, USA, and The Macula Foundation, Inc., New York, NY, USA. The funding organizations had no role in the design or conduct of this research.

\section{Compliance with ethical guidelines}

\section{Competing interests}

K. Bailey Freund: Genentech: Consultant, ThromboGenics: Consultant, Ohr Pharmaceutical: Consultant, Bayer HealthCare: Consultant, Heidelberg Engineering: Consultant. There are no other relevant disclosures.

Received: 17 July 2015 Accepted: 11 August 2015

Published online: 01 September 2015

\section{References}

1. Zamir E, Kaiserman I, Chowers I. Laser pointer maculopathy. Am J Ophthalmol. 1999;127:728-9.

2. Lally DR, Duker JS. Foveal injury from a red laser pointer. JAMA Ophthalmol. 2014;132:297.

3. Bhavsar KV, Wilson D, Margolis R, et al. Multimodal imaging in handheld laser-induced maculopathy. Am J Ophthalmol. 2015;159(227-231):e222

4. Lee GD, Baumal CR, Lally D, Pitcher JD, Vander J, Duker JS. Retinal injury after inadvertent handheld laser exposure. Retina. 2014;34:2388-96.

5. Luttrull JK, Hallisey J. Laser pointer-induced macular injury. Am J Ophthalmol. 1999;127:95-6.

6. Rusu I, Sherman J, Gallego-Pinazo R, Lam M, Freund KB. Spectral-domain optical coherence tomography and fundus autofluorescence findings in a case of laser pointer-induced maculopathy. Retin Cases Brief Rep. 2013;7:371-5

7. Wyrsch S, Baenninger PB, Schmid MK. Retinal injuries from a handheld laser pointer. N Engl J Med. 2010;363:1089-91.

8. Ziahosseini K, Doris JP, Turner GS (2010) Laser eye injuries. Maculopathy from handheld green diode laser pointer. BMJ 340:c2982.

\section{Submit your next manuscript to BioMed Central and take full advantage of:}

- Convenient online submission

- Thorough peer review

- No space constraints or color figure charges

- Immediate publication on acceptance

- Inclusion in PubMed, CAS, Scopus and Google Scholar

- Research which is freely available for redistribution

Submit your manuscript at www.biomedcentral.com/submit 